\title{
FIXED POINT FREE INVOLUTIONS AND EQUIVARIANT MAPS ${ }^{1}$
}

\author{
P. E. CONNER AND E. E. FLOYD
}

1. Preliminaries. We are concerned with involutions without fixed points, together with equivariant maps connecting such involutions. An involution $T$ is a homeomorphism of period 2 of a Hausdorff space $X$ onto itself; that is, $T^{2}(x)=x$ for all $x \in X$.

There is associated with an involution $T$ on $X$ the orbit space $X / T$, obtained by identifying $x$ with $T(x)$ for all $x \in X$. Denote by $\nu: X \rightarrow X / T$ the decomposition map. If $T$ is fixed point free, then $\nu: X \rightarrow X / T$ is a local homeomorphism. The map $\nu$ is always both open and closed.

In addition to fixed point free involutions we also study equivariant maps. If spaces $X, X^{\prime}$ carry involutions $T, T^{\prime}$ respectively, then a map $m: X \rightarrow X^{\prime}$ is equivariant provided that $m(T(x))=T^{\prime}(m(x))$ for all $x \in X$. An equivariant map $m: X \rightarrow X^{\prime}$ induces a map $M: X / T$ $\rightarrow X^{\prime} / T^{\prime}$.

The most fundamental involution without fixed points is the antipodal involution $A$ on the $n$-sphere $S^{n}$, given by $A\left(x_{1}, \cdots, x_{n+1}\right)$ $=\left(-x_{1}, \cdots,-x_{n+1}\right)$. When we speak of $S^{n}$ as carrying a fixed point free involution, it is to be understood that we refer to $A$. For $S^{n}$, we have the classical body of results of Lyusternik-Schnirelmann and Borsuk-Ulam. Some of these well-known results are summarized in the following.

(1.1) The following are true for every $n$ :

(i) there is no equivariant map of $S^{n+1}$ into $S^{n}$;

(ii) every equivariant map of $S^{n}$ into itself is of odd degree; in particular, it is essential;

(iii) the Lyusternik-Schnirelmann category of $S^{n} / A=P^{n}$ is $n$;

(iv) for every covering of $S^{n}$ by $n+1$ closed sets $A_{1}, \cdots, A_{n+1}$, some set $A_{i}$ contains an antipodal pair;

(v) for every map $f: S^{n} \rightarrow R^{n}$ there is a point $x \in S^{n}$ for which $f(x)$ $=f(A(x))$.

An address delivered before the East Lansing meeting of the Society by P. E. Conner under the title Involutions and equivariant maps, on September 2, 1960, by invitation of the Committee to Select Hour Speakers for Summer and Annual Meetings; received by the editors June 24, 1960.

1 This research was supported by the United States Air Force through the Air Force Office of Scientific Research of the Air Research and Development Command, under contract AF 49(638)-72 at the University of Virginia. 
(vi) the cohomology ring $H^{*}\left(P^{n} ; Z_{2}\right)$ is isomorphic to the truncated polynomial ring $Z_{2}(x) /\left[x^{n+1}\right]$.

Here cat $X$ is one less than the least number of closed sets required to cover $X$ so that each set is contractible to a point in $X$. By $Z_{2}(x) /\left[x^{n+1}\right]$ we mean the polynomial ring $Z_{2}(x)$ factored by the ideal which $x^{n+1}$ generates.

The following two questions are suggested by (1.1) for a given fixed point free involution $T: X \rightarrow X$.

(1) For which integer $n$ is there an equivariant map of $S^{n}$ into $X$, but no equivariant map of $S^{n+1}$ into $X$ ?

(2) For which integer $n$ is there an equivariant map of $X$ into $S^{n}$, but not of $X$ into $S^{n-1}$ ?

In view of (1.1), the answer to both questions for $X=S^{n}$ is $n$. It appears to us that the above are the most fundamental questions for a fixed point free involution; we have first realized the importance of (2) from the work of Yang [17] on mappings of spheres. We do not try for a really general answer to either question, but are able to make a computation in several classes of examples.

The results (1.1) also suggest a related problem; namely for which fixed point free involutions $T: X \rightarrow X$ are each of the following pairs of statements equivalent.

I. (a) There is an equivariant map $S^{n} \rightarrow X$, but no equivariant $\operatorname{map} S^{n+1} \rightarrow X$.

$\left(a^{\prime}\right)$ There is an equivariant map $S^{n} \rightarrow X$, and every such equivariant map is essential.

II. (b) There is an equivariant map $X \rightarrow S^{n}$, but no equivariant map $X \rightarrow S^{n-1}$.

$\left(b^{\prime}\right)$ There is an equivariant map $X \rightarrow S^{n}$, and every such equivariant map is essential.

Some thought reveals that in fact the statements $(a)$ and $\left(a^{\prime}\right)$ are always equivalent. Furthermore, it is true that $\left(b^{\prime}\right)$ implies $(b)$ for all $X$. However, the question of whether (b) implies $\left(b^{\prime}\right)$ is much more interesting. We shall see that (b) does not always imply $\left(b^{\prime}\right)$. We obtain conditions under which (b) implies $\left(b^{\prime}\right)$ (for example, if $\operatorname{dim} X \leqq 2 n-3)$, and also give examples where the implication is false (in fact, one with $\operatorname{dim} X=2 n-2$ ).

We have by no means exhausted the list of questions suggested by (1.1). If we examine statements (iv) and (vi) we might guess that for a general fixed point free involution the answers to questions (1) and (2) are related to cat $X / T$ and to the cohomology ring $H^{*}\left(X / T ; Z_{2}\right)$. We shall make these relations clear in later sections.

Our interest in these matters was stimulated by the classical re- 
sults of Lyusternik-Schnirelmann and Borsuk-Ulam, together with the more recent works of Yang $[17 ; 18 ; 19]$ and of Bourgin [3]. The results of $\$ 5$ on stability of equivariant homotopy classes were inspired by the Spanier-Whitehead $S$-category $[12 ; 13]$.

2. Equivariant maps. In this section, $A$ will denote a fixed point free involution on a finite cell complex $K$, with $A$ also cellular. Consider also an involution $T: X \rightarrow X$, not necessarily fixed point free in this section. Denote by $\nu: K \rightarrow K / A$ the cellular map of $K$ onto the orbit space $K / A$. Denote by $E(K, X)$ the collection of all equivariant maps $m: K \rightarrow X$. If $T$ has fixed points, $E(K, X)$ is nonempty, since we may map all of $K$ into a fixed point of $X$; however if $T$ has no fixed points, $E(K, X)$ may of course be empty. We give $E(K, X)$ the compact-open topology. Two maps $m_{0}, m_{1} \in E(K, X)$ are equivariantly homotopic if and only if there is a continuous 1-parameter family of maps $m_{r} \in E(K, X)$ joining $m_{0}$ to $m_{1}$. Equivariant homotopy is an equivalence relation, and the collection of equivariant homotopy classes in $E(K, X)$ is denoted by $E_{*}(K, X)$. We understand that $E_{*}(K, X)=\varnothing$ if and only if $E(K, X)=\varnothing$.

The study of $E(K, X)$ and $E_{*}(K, X)$ is greatly simplified by the important observation of Heller [7] that the equivariant maps of $K$ into $X$ may be identified with the cross-sections of an appropriate fibre bundle. This bundle is constructed by first forming the product $K \times X$ and introducing a diagonal involution $T^{\prime}=A \times T$ on $K \times X$ by $T^{\prime}(k, x)=(A(k), T(x))$. The projection map $p: K \times X \rightarrow K$ is an equivariant map, thus it defines a map $\alpha:(K \times X) / T^{\prime} \rightarrow K / A$. Since $A$ has no fixed points, $\alpha$ is a locally trivial fibre map over $K / A$ with fibre $X$ and structural group $Z_{2}$. Consider the commutative diagram

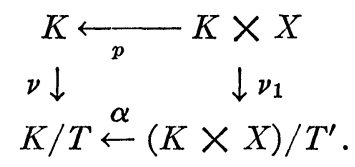

For each $m$ in $E(K, X)$, define a cross-section $s: K / A \rightarrow(K \times X) / T^{\prime}$ by

$$
s(\nu(k))=\nu_{1}(k, m(k))
$$

for all $k \in K$. Note that

$$
\begin{aligned}
s(\nu(A(k))) & =\nu_{1}(A(k), m(A(k)))=\nu_{1}(A(k), T m(k)) \\
& =\nu_{1}\left(T^{\prime}(k, m(k))\right)=\nu_{1}(k, m(k))=s(\nu(k)) .
\end{aligned}
$$

Thus the cross-section $s$ is well defined. Conversely let $s: K / A$ $\rightarrow(K \times X) / T^{\prime}$ be a cross-section. Since $A$ has no fixed points, there 
is for each $k \in K$ a unique point $m(k) \in X$ such that $s(\nu(k))=\nu_{1}(k, m(k))$. To see that $m$ is equivariant we write

$$
s(\nu(A(k)))=\nu_{1}(A(k), m(A(k)))=s(\nu(k))=\nu_{1}(k, m(k))
$$

which implies

$$
T^{\prime}(k, m(k))=(A(k), T m(k))=(A(k), m(A(k))) .
$$

This correspondence between $E(K, X)$ and the cross-sections of $\alpha:(K \times X) / T^{\prime} \rightarrow K / A$ carries equivariant homotopies into homotopies of cross-sections. This observation of Heller's means that in studying $E(K, X)$ and $E_{*}(K, X)$ we may use the well known methods of obstruction theory for the study of cross-sections of a fibre bundle. For example, in view of 37.5 in Steenrod [14], we have an equivariant Hopf classification theorem.

(2.1) Let $A$ be a fixed point free involution on a finite cell complex $K$ of dimension $n$, and let $T$ be an involution on an $(n-1)$-connected space $X, n \geqq 1$. Then there is a one-to-one correspondence between $E_{*}(K, X)$ and $H^{n}\left(K / A ; B\left(\pi_{n}(X)\right)\right)$.

If $n=1$, we must assume $\pi_{1}(X)$ abelian. In addition to (2.1), we also have an important deformation theorem. If $Y \subset X$ is a closed subset of $X$, then $\pi_{i}(X, Y)=0,0 \leqq i \leqq n$, will signify that $X$ and $Y$ are pathwise connected, that $\pi_{1}(X)=\pi_{1}(Y)=0$, and that the group $\pi_{i}(X, Y)$ is trivial for $2 \leqq i \leqq n$.

(2.2) Let $A$ be a fixed point free involution on a finite cell complex $K$. Let $T$ be an involution on a space $X$, and let $Y$ be a closed invariant subset of $X$ such that $\pi_{i}(X, Y)=0,0 \leqq i \leqq n$. If $\operatorname{dim} K<n$, the natural correspondence $j_{*}: E_{*}(K, Y) \rightarrow E_{*}(K, X)$ is one-to-one and onto. If $\operatorname{dim} K$ $=n, j_{*}$ is onto.

A brief but clear discussion of the necessary deformation theorems is found in $\mathrm{Hu}$ [9]. In particular, note that under the hypothesis $\operatorname{dim} K=n$ in (2.2), $E(K, Y)=\varnothing$ if and only if $E(K, X)=\varnothing$.

Consider now the suspension $S(K)$ of $K$. That is, $S(K)$ is formed from $K \times I$ by collapsing $K \times 0$ and $K \times 1$ each to a point; the point coming from $K \times 0$ we call the lower vertex of $S(K)$. We may consider $K$ as embedded in $S(K)$ by identifying $K$ with $K \times(1 / 2)$. If $A$ is an involution on $K$, there is a suspended involution on $S(K)$ generated by $(k, t) \rightarrow(A(k), 1-t)$. This involution is an extension of $A$ to an involution on $S(K)$; we denote the extension by $A$ also.

Select now a base point $x_{0} \in X$. There is the collection $E\left(S(K), X ; x_{0}\right)$ of equivariant maps $S(K) \rightarrow X$ which map the lower vertex of $S(K)$ into $x_{0}$, and the collection $E\left(S(K), X ; x_{0}\right)$ of equivariant homotopy classes of $E\left(S(K), X ; x_{0}\right)$. There is a natural correspondence 
$j_{*}: E_{*}\left(S(K), \quad X ; x_{0}\right) \rightarrow E_{*}(S(K), X)$ induced by the inclusion $E\left(S(K), X ; x_{0}\right) \subset E(S(K), X)$.

(2.3) If $T$ is an involution on a pathwise connected space $X$, then for any $x_{0} \in X, j_{*}: E_{*}\left(S(K), X ; x_{0}\right) \rightarrow E_{*}(S(K), X)$ is onto. If in addition $\pi_{1}(X)=0$ then $j_{*}$ is also one-to-one.

We now introduce the space $P_{x_{0}}=P_{x_{0}}(X)$, the space of all paths in $X$ joining $x_{0}$ to $T\left(x_{0}\right)$, with the compact-open topology. Note that possibly $P_{x_{0}}=\varnothing$ if $X$ is not arcwise connected. We place an involution $\Gamma$ on $P_{x_{0}}$ by

$$
\Gamma(p)(t)=T(p(1-t)), \quad p \in P_{x_{0}} .
$$

(2.4) For any point $x_{0} \in X$, there is a natural one-to-one correspondence between $E_{*}\left(K, P_{x_{0}}\right)$ and $E_{*}\left(S(K), X ; x_{0}\right)$.

This is easily seen; in fact, the correspondence is actually a homeomorphism. With these facts, we can now prove an equivariant suspension theorem. The object is to relate $E_{*}(K, X)$ to $E_{*}(S(K), S(X))$, for $X$ compact. It will be noted that the usual suspension of a map in $E(K, X)$ is still an equivariant map, thus there is a suspension correspondence $s_{*}: E(K, X) \rightarrow E(S(K), S(X))$.

(2.5) Theorem. Let $A$ be a fixed point free involution on a finite cell complex $K$, and let $T$ be an involution on a compact $(n-1)$-connected space $X, n \geqq 2$. If $\operatorname{dim} K<2 n-1$, then $s_{*}: E_{*}(K, X) \rightarrow E_{*}(S(K), S(X))$ is a one-to-one correspondence. If $\operatorname{dim} K=2 n-1$, then $s_{*}$ is onto.

The suspension $S(X)$ is $n$-connected. Choose $x_{0} \in S(X)$ to be the lower vertex; then $j_{*}: E_{*}\left(S(K), S(X) ; x_{0}\right) \rightarrow E_{*}(S(K), S(X))$ is a oneto-one correspondence between the two. By (2.4) we may replace $E_{*}\left(S(K), S(X) ; x_{0}\right)$ by $E_{*}\left(K, P_{x_{0}}(S(X))\right)$. There is the equivariant embedding $X \subset P_{x_{0}}(S(X))$ for which, as is well-known [13], $\pi_{i}\left(P_{x_{0}}, X\right)$ $=0$ for $0 \leqq i \leqq 2 n-1$. We complete the argument by applying (2.2).

We note that under the hypotheses of (2.5) if $\operatorname{dim} K \leqq 2 n-1$, then $E_{*}(S(K), S(X))=\varnothing$ if and only if $E_{*}(K, X)=\varnothing$.

$\$ 2$ has consisted of general results needed for our principal lines of inquiry. We would like to know additional general facts about the space $E(K, X)$ and the set $E_{*}(K, X)$, but turn instead to the special cases $E\left(S^{n}, X\right)$ and $E\left(X, S_{n}\right)$ under the assumption that $X$ is fixed point free.

3. The index and the co-index. In this section we shall deal only with fixed point free involutions. Our discussion suggests the definition of two functions on the class of fixed point free involutions.

(3.1) Definition. Let $T$ denote a fixed point free involution on a 
space $X$. The index of $(T, X)$ is the largest integer $n$ for which there is an equivariant map of $S^{n}$ into $X$. The co-index of $(T, X)$ is the least integer $n$ for which there is an equivariant map of $X$ into $S^{n}$.

We abbreviate index and co-index by ind $X$ and co-ind $X$ respectively. Write ind $X=-1$, or co-ind $X=-1$, if and only if $X=\varnothing$. Observe that if $X \neq \varnothing$ then $S^{0}$ can always be equivariantly mapped into $X$. It may happen for a particular $X$ that there is no upper bound on the dimension of the sphere which can be equivariantly mapped into $X$; then we write ind $X=\infty$. Also if $X$ cannot be equivariantly mapped into $S^{n}$ no matter how large $n$, write co-ind $X=\infty$. The coindex function is precisely Yang's $B$-index [17]. The following remark is an immediate corollary of the definition.

(3.2) Let $T$ and $T^{\prime}$ be fixed point free involutions on spaces $X, Y$ respectively, and let $m: X \rightarrow Y$ be equivariant. Then

$$
\text { ind } X \leqq \text { ind } Y \text {, co-ind } X \leqq \text { co-ind } Y \text {. }
$$

Thus, an equivariant map decreases neither index nor co-index. It follows from (1.1) that ind $S^{n}=$ co-ind $S^{n}=n$. From this we derive

(3.3) For any fixed point free involution $T$ on a space $X$, ind $X$ $\leqq$ co-ind $X$.

We now begin some remarks about co-index. An invariant subset $A \subset X$ admits a cross-section if and only if there is a set $B \subset A$ which is closed in $A$ and such that $A=B \cup T(B), B \cap T(B)=\varnothing$. If $\nu: X$ $\rightarrow X / T$ denotes the orbit map, then $B \subset X / T$ admits a cross-section if and only if there is a map $s: B \rightarrow X$ with $\nu s$ the identity. The two definitions are easily seen to be equivalent in the sense that $B \subset X / T$ admits a cross-section if and only if the invariant set $\nu^{-1}(B)=A \subset X$ admits a cross-section. There are the following characterizations of co-index, suggested by Yang's results [17] on $B$-index.

(3.4) Theorem. If $T$ is a fixed point free involution on a normal space $X$, then the following are equivalent:

(1) $X$ can be covered by $n+1$ closed sets $A_{1}, \cdots, A_{n+1}$ such that each $A_{i}$ is invariant and admits a cross-section;

(2) $X$ admits a finite covering of order $\leqq n$ by closed invariant sets each of which admits a cross-section;

(3) $X$ admits a covering by $n+1$ open invariant sets $W_{1}, \cdots, W_{n+1}$, each of which admits a cross-section;

(4) co-ind $X \leqq n$;

(5) there is a fixed point free involution $T^{\prime}$ on a paracompact space $Y$, with cat $Y / T^{\prime} \leqq n$, and an equivariant map $m: X \rightarrow Y$.

As a corollary of (3.3) and (3.4) we have: 
(3.5) If $T$ is a fixed point free involution on a paracompact space $X$, then

$$
\text { ind } X \leqq \text { co-ind } X \leqq \text { cat } X / T \text {. }
$$

The next lemma, used in proving (3.4) and (3.7), is purely settheoretic in nature.

(3.6) If $U$ is a locally finite open covering of order $\leqq n$ on a normal space $X$, then $X$ can be covered by $n+1$ open sets $W_{1}, \cdots, W_{n+1}$, each of which can be written as the disjoint union of open sets each contained in some element of $U$.

If the elements of $U$ are invariant under an involution on $X$, then the $W_{i}$ may be assumed invariant, and furthermore $W_{i}$ may be represented as the disjoint union of open invariant sets each of which belong to some element of $U$. A point to be observed here is that if each of a collection of disjoint open invariant sets admits a crosssection, then so does the union. It should also be noted that throughout we agree that dimension refers to covering dimension defined in terms of arbitrary open coverings.

(3.7) If $T$ is a fixed point free involution on a paracompact space $X$, then

$$
\text { ind } X \leqq \operatorname{co-ind} X \leqq \operatorname{dim} X / T \text {. }
$$

In the proof, we may as well assume $\operatorname{dim} X / T=n<\infty$. Since $\nu$ is a local homeomorphism, there is an open covering $V$ of $X / T$ each element of which admits a cross-section. We may as well assume $V$ locally finite. Since $\operatorname{dim} X / T=n$ there is an open covering $V^{\prime}$ of order $\leqq n$ refining $V$. Let $\{\alpha\},\{\beta\}$ respectively denote the indexing sets of $V^{\prime}$ and $V$ and let $h:\{\alpha\} \rightarrow\{\beta\}$ be a function chosen so that $V_{\alpha}^{\prime} \subset V_{k(\alpha)}$ for all $\alpha \in\{\alpha\}$. Let $U_{\beta}=\bigcup_{h(\alpha)=\beta} V_{\alpha}^{\prime}$. Then $U=\left\{U_{\beta}\right\}$ is a locally finite open covering of order $\leqq n$ each element of which admits a cross-section. We apply (3.6) to $U$ to conclude that $X / T$ is covered by $n+1$ open sets $W_{1}, \cdots, W_{n+1}$ each of which admits a cross-section. The open invariant sets $\nu^{-1}\left(W_{i}\right)$ cover $X$ and each admits a crosssection, so from (3.3) we have co-ind $X \leqq n=\operatorname{dim} X / T$.

If $X$ is a separable metric space, then it is well-known that $\operatorname{dim} X / T$ $=\operatorname{dim} X$. Actually (3.7) can be strengthened considerably.

(3.8) THEOREM. If $T$ is an involution on a finite dimensional separable metric space $X$, and if $H^{i}(X ; Z)=0$ for all $i \geqq k$, then co-ind $X \leqq k$.

The cohomology groups are here the Alexander-Spanier cohomology groups with integral coefficients. The proof is too long to be included in this outline.

Now we shall turn to the index. We may think of the computation 
of index as a problem in obstruction theory of the following type. Let $W=\bigcup_{n=0}^{\infty} S^{n}$ denote the union of the ascending chain of spheres $S^{0} \subset S^{1} \subset \cdots \subset S^{n} \subset \ldots$; there is an obvious antipodal map $A$ on $W$. If $W$ is given the $C W$-topology, $A$ is a fixed point free involution on an acyclic complex $W$. The $n$-skeleton of $W$ is $S^{n}$. Let $P^{\infty}=W / A$ denote the quotient space; as usual, we refer to $P^{\infty}$ as the infinite dimensional real projective space. The $n$-skeleton of $P^{\infty}$ is $P^{n}$. Consider the fibre bundle $\alpha:(W \times X) / T^{\prime} \rightarrow W / A=P^{\infty}$. An equivariant map $m \in E\left(S^{n}, X\right)$ corresponds to a partial cross-section defined over the $n$-skeleton of $P^{\infty}$. If $X$ is $n$-simple then we may associate with $m$ an obstruction class $k(m) \in H^{n+1}\left(P^{\infty} ; B\left(\pi_{n}(X)\right)\right)$. Note that $E\left(S^{n+1}, X\right)$ $\neq \varnothing$ if and only if for some map $m \in E\left(S^{n}, X\right), k(m)=0$ in $H^{n+1}\left(P^{\infty} ; B\left(\pi_{n}(X)\right)\right)$. Of course $k(m)$ depends only on the equivariant homotopy class of $m$, so that we have a natural correspondence

$$
k_{*}: E_{*}\left(S^{n}, X\right) \rightarrow H^{n+1}\left(P^{\infty} ; B\left(\pi_{n}(X)\right)\right) .
$$

The reader may have noted that if $X$ is $(n-1)$-connected, then the image of $k_{*}: E_{*}\left(S^{n}, X\right) \rightarrow H^{n+1}\left(P^{\infty} ; B\left(\pi_{n}(X)\right)\right)$ consists of exactly one element [14]. The element is called the Eilenberg-MacLane $k$-invariant of $X[4$, p. 361].

The group $H^{n+1}\left(P^{\infty} ; B\left(\pi_{n}(X)\right)\right)$ may be thought of as the cohomology groups of the discrete group $Z_{2}$ with coefficients in the $Z\left(Z_{2}\right)$ module $\pi_{n}(X)$ [4]. The involution $T$ defines an automorphism $T_{*}: \pi_{n}(X) \rightarrow \pi_{n}(X)$ of period 2 since $X$ is $n$-simple. Thus $Z_{2}$ acts as a group of automorphisms on $\pi_{n}(X)$. In this way, $H^{n+1}\left(Z_{2} ; \pi_{n}(X)\right)$ is defined; hereafter we use it to replace $H^{n+1}\left(P^{\infty} ; B\left(\pi_{n}(X)\right)\right)$. The computation for $H^{n+1}\left(Z_{2} ; \pi_{n}(X)\right)$ is as follows:

Let

$$
\begin{aligned}
I_{n} & =\left\{\sigma: \sigma \in \pi_{n}(X), T_{*}(\sigma)=(-1)^{n+1} \sigma\right\}, \\
N_{n} & =\left\{\sigma+(-1)^{n+1} T_{*}(\sigma): \sigma \in \pi_{n}(X)\right\} ;
\end{aligned}
$$

then $H^{n+1}\left(Z_{2} ; \pi_{n}(X)\right)=I_{n} / N_{n}[4$, p. 250].

(3.9) Let $T$ be a fixed point free involution on an $n$-simple space $X$. The following are equivalent if ind $X \geqq n$ :

(1) $E\left(S^{n+1}, X\right) \neq \varnothing$;

(2) some map in $E\left(S^{n}, X\right)$ is null-homotopic;

(3) for some map $m \in E_{*}\left(S^{n}, X\right), k_{*}(m)=0$ in $H^{n+1}\left(Z_{2} ; \pi_{n}(X)\right)$;

(4) ind $X \geqq n+1$.

In particular if $X$ is $(n-1)$-connected, then ind $X \geqq n$. Moreover ind $X \geqq n+1$ if and only if the $k$-invariant $k^{n+1}$ is zero.

(3.10) Theorem. Let $f: X \rightarrow Y$ be an equivariant map, where $X$ and 
$Y$ carry fixed point free involutions and ind $X=$ ind $Y=n<\infty$. If $X$ and $Y$ are $n$-simple, then

$$
f_{*}: H^{n+1}\left(Z_{2} ; \pi_{n}(X)\right) \rightarrow H^{n+1}\left(Z_{2} ; \pi_{n}(Y)\right)
$$

is nontrivial.

An immediate corollary follows if we apply (3.10) to the identity map of $X$.

(3.11) Let $T$ be a fixed point free involution on $X$, and suppose ind $X=n<\infty$. If $X$ is $n$-simple, then $H^{n+1}\left(Z_{2} ; \pi_{n}(X)\right) \neq 0$.

Referring to the pair of statements labelled $I$ in the first section, it will be noted that (3.9), (3.10), and (3.11) are all based on the fact that the pair of statements are equivalent. We mentioned, however, that the pair labelled II are not generally equivalent. Thus we cannot expect to have such strong results for co-index. We now give our best result parallel to (3.11).

(3.12) Theorem. Let $A$ be a cellular involution without fixed points on a finite cell complex $K$, where $n=$ co-ind $K$. If $\operatorname{dim} K \leqq 2 n-3$ then $\pi^{n}(K) \neq 0$. In fact, every equivariant map $f: K \rightarrow S^{n}$ is essential. If $n=1,2$ the conclusions are true without dimensional restrictions on $K$.

Proof. We prove the assertions for $n \geqq 3$, leaving the assertions $n=1,2$ to a later section. We may apply (2.5), using $X=S^{n-1}$. Since

$$
\operatorname{dim} K \leqq 2 n-3=2(n-1)-1
$$

it follows that $s_{*}: E_{*}\left(K, S^{n-1}\right) \rightarrow E_{*}\left(S(K), S^{n}\right)$ is onto. However, $E_{*}\left(K, S^{n-1}\right)=\varnothing$ by hypothesis, so that $E_{*}\left(S(K), S^{n-1}\right)=\varnothing$ also. If some map in $E\left(K, S^{n}\right)$ were inessential, it could be extended to an equivariant $S(K) \rightarrow S^{n}$, so that $E\left(S(K), S^{n}\right) \neq \varnothing$. The conclusion follows.

It is reasonable to suppose that under the hypotheses of (3.12) that $H_{n}\left(Z_{2} ; \pi^{n}(K)\right) \neq 0$, but we do not know if this is true.

Under certain circumstances, then, the pair of statements labelled II are equivalent. Naturally we now seek examples when the pair are not equivalent. Recall the space $P_{x_{0}}(X)$ of $\S 2$, with its involution $\Gamma$. We consider now $P_{x_{0}}\left(S^{n}\right)$, where $x_{0} \in S^{n}$.

(3.13) Theorem. Let $T$ be a fixed point free involution on a space $X$. There exists an inessential equivariant map $m: X \rightarrow S^{n}$ if and only if there exists an equivariant map $m^{\prime}: X \rightarrow P_{x_{0}}\left(S^{n}\right)$.

Consider the map $\omega: P_{x_{0}} \rightarrow S^{n}$ mapping a path $p$ into $\omega(p)=p(1 / 2)$. Now $\omega$ is equivariant. It is also inessential with the contracting homotopy $\omega_{t}(p)=p(t / 2)$. If there exists an equivariant map $m^{\prime}: X$ 
$\rightarrow P_{x_{0}}\left(S^{n}\right)$, then the equivariant map $\omega m^{\prime}: X \rightarrow S^{n}$ is inessential. We omit the proof of the converse.

We shall now show that for $n$ odd and $\geqq 3$, co-ind $P_{x_{0}}\left(S^{n}\right)=n$. In the following observation, call an element $\gamma \in H^{n}(X ; Z)$ a spherical cohomology class if there is a map $f: X \rightarrow S^{n}$ with $\gamma$ in the image of $f^{*}: H^{n}\left(S^{n} ; Z\right) \rightarrow H^{n}(X ; Z)$.

(3.14) If $T: X \rightarrow X$ is an involution without fixed points for which ind $X=$ co-ind $X=n$, then $H_{n}(X ; Z)$ contains a spherical homology class, of infinite order, not divisible by two, and $H^{n}(X ; Z)$ contains a spherical cohomology class of infinite order and not divisible by two.

To see the remark concerning homology, suppose $f: S^{n} \rightarrow X$ and $g: X \rightarrow S^{n}$ are equivariant. Then $g f: S^{n} \rightarrow S^{n}$ is equivariant and hence of odd degree. Then $f_{*}(\gamma), \gamma$ a generator of $H_{n}\left(S^{n} ; Z\right)$ is the desired element. Note that homology and cohomology in (3.14) refers to the singular groups.

We now prove that for $n$ odd and $\geqq 3$, co-ind $P_{x_{0}}\left(S^{n}\right)=n$. We found an equivariant map $P_{x_{0}} \rightarrow S^{n}$; hence co-ind $P_{x_{0}} \leqq n$. There is an equivariant embedding $S^{n-1} \subset P_{x_{0}}$. Hence

$$
n-1 \leqq \text { ind } P_{x_{0}} \leqq \text { co-ind } P_{x_{0}} \leqq n .
$$

Suppose that

$$
n-1=\text { ind } P_{x_{0}}=\text { co-ind } P_{x_{0}} .
$$

According to (3.14), $H^{n-1}\left(P_{x_{0}}\left(S^{n}\right) ; Z\right)$ then contains a nontrivial spherical class. Of course $P_{x_{0}}\left(S^{n}\right)$ has the homotopy type of the loop space $\Omega\left(S^{n}\right)$; for $n$ odd, Serre has shown that for every $h \in H^{n-1}\left(\Omega\left(S^{n}\right) ; Z\right)$ with $h \neq 0$ we have $h^{2} \neq 0$. Hence there exist no nontrivial spherical classes in $H^{n-1}\left(P_{x_{0}} ; Z\right)$. We conclude, then, that for $n$ odd and $\geqq 3$,

$$
n-1=\text { ind } P_{x_{0}}\left(S^{n}\right)<\text { co-ind } P_{x_{0}}\left(S^{n}\right)=n .
$$

Thus co-ind $P_{x_{0}}=n$, but there is an inessential equivariant map $P_{x_{0}} \rightarrow S^{n}$. This example shows that the pair of statements labelled II are not always equivalent. Incidentally we also have a fixed point free involution for which index and co-index differ. If the integer $n$ is even and $>2$ we do not know whether co-ind $P_{x_{0}}=n$ or $n-1$.

We make now a few remarks concerning the Stiefel manifold $V_{n, 2}$, the set of all orthogonal 2-frames in $R^{n}$. There are several fixed point free involutions on $V_{n, 2}$; for example we may send a 2 -frame $\left(v_{1}, v_{2}\right)$ into $\left(v_{1},-v_{2}\right)$, or into $\left(-v_{1},-v_{2}\right)$, or into $\left(v_{2}, v_{1}\right)$.

(3.15) Let $T$ be a fixed point free involution on $V_{n, 2}$. If $n$ is odd, then

$$
n-2 \leqq \text { ind } V_{n, 2}<\text { co-ind } V_{n, 2} \text {. }
$$


For $n$ odd the integral homology groups of $V_{n, 2}$ are $H_{n-2}\left(V_{n, 2}\right)$ $\approx Z_{2}, H_{2 n-3}\left(V_{n, 2}\right) \approx Z$ and $H_{i}=0$ for other $i>0$. In view of (3.9), $n-2 \leqq$ ind $V_{n, 2}$. Suppose ind $V_{n, 2}=$ co-ind $V_{n, 2}$. By (3.14), ind $V_{n, 2}$ would be either $n-2$ or $2 n-3$ and (3.14) also rules out $n-2$. Thus ind $V_{n, 2}=2 n-3=\operatorname{dim} V_{n, 2}$. Then the fundamental class in $H_{2 n-3}\left(V_{n, 2} ; Z_{2}\right)$ would be a mod 2 spherical homology class. But for manifolds this can only be true for $\bmod 2$ homology spheres. Thus

$$
n-2 \leqq \text { ind } V_{n, 2}<\text { co-ind } V_{n, 2} \text {. }
$$

The involution $\left(v_{1}, v_{2}\right) \rightarrow\left(v_{1},-v_{2}\right)$ can be mapped equivariantly into $S^{n-1}$ by projection on the second factor. For this involution co-ind $V_{n, 2}$ $\leqq n-1$. For $n$ odd we then have

$$
n-2=\text { ind } V_{n, 2}<\text { co-ind } V_{n, 2}=n-1 .
$$

The same result is true, for $n$ odd, for the involutions $\left(v_{1}, v_{2}\right)$ $\rightarrow\left(-v_{1},-v_{2}\right)$ and $\left(v_{1}, v_{2}\right) \rightarrow\left(v_{2}, v_{1}\right)$. Definitive results for $V_{n, 2}, n$ even, appear very difficult.

To conclude the section, consider the much more general case of a compact Lie group $G$ operating on a Hausdorff space $X$; of course, an involution corresponds precisely to the case $G=Z_{2}$. Suppose also that $G$ operates freely on $X$; that is, if $g \neq e$ then $g: X \rightarrow X$ has no fixed points. In particular, there is the $(n+1)$-fold join $G^{(n)}=G \circ G \circ \ldots$ $\circ G ; G$ operates in the usual diagonal fashion on $G^{(n)}$. Note that for $G=Z_{2}$ that $G^{(n)}=S^{n}$. This suggests the following definition for ind $X$ and co-ind $X$. Namely, ind $X$ is the largest $n$ for which there exists an equivariant map $G^{(n)} \rightarrow X$ and co-ind $X$ is the largest $n$ for which there exists an equivariant $X \rightarrow G^{(n)}$. With these definitions, several of the theorems of this section can be extended to this more general setting.

4. Cohomology co-index. We have raised the question of when there exist equivariant maps $K \rightarrow X$. It is natural to consider the purely homological criteria that must be satisfied for this to be the case. In particular we come to characteristic classes.

The best known and most easily managed such class comes from cohomology with coefficients $Z_{2}$. If $T$ is a fixed point free involution on a paracompact space $X$, there is the fundamental class $c=c(X)$ $\in H^{1}\left(X / T ; Z_{2}\right)$ of the involution. For example, $c$ can be considered as the Whitney class $w_{1}$ of the 0 -sphere bundle $\nu: X \rightarrow X / T$. If $m: X \rightarrow Y$ is equivariant then $M^{*}: H^{1}(Y / T) \rightarrow H^{1}(X / t)$ maps $c(Y)$ onto $c(X)$. In particular if the $m$ th power $c^{m}(X)$ is non-zero, so also is $c^{m}(Y)$. Thus we have a strong necessary condition which equivariant images $Y$ of $X$ must satisfy. 
For later purposes, we define $c$ in terms of the Smith-Gysin sequence (coefficients $Z_{2}$ ):

$$
\cdots H^{0}(X / T) \stackrel{\delta^{*}}{\rightarrow} H^{1}(X / T) \stackrel{\nu^{*}}{\rightarrow} H^{1}(X) \stackrel{\tau^{*}}{\rightarrow} H^{1}(X / T) \stackrel{\delta^{*}}{\rightarrow} \cdots .
$$

Here the cohomology is taken to be AWS-cohomology. If $\epsilon$ denotes the unit class of $H^{0}(X / T)$, then $c=\delta^{*}(\epsilon)$. More generally, $\delta^{*}: H^{n}(X / T)$ $\rightarrow H^{n+1}(X / T)$ is given by $\delta^{*}(\alpha)=\alpha \cdot c$. There are the powers $c^{m}$ $\in H^{m}(X / T)$, and of course $c^{m} \cdot c^{n}=c^{m+n}$.

The above is, of course, very well known. For involutions, such methods have been used very successfully by Bourgin [3] and Yang [18]. We shall need a similar technique with coefficients $Z$. While we are about it, we define fundamental classes for any finite group $G$ and any principal ideal domain $L$.

First, we use the equivariant cohomology theory $[5 ; 6]$. Let a finite group $G$ operate freely on a paracompact space $X$, and let $J$ be a $L(G)$-module. An equivariant cochain is then an AlexanderSpanier cochain $\phi$, assigning to each $(n+1)$-tuple $x_{0}, x_{1}, \cdots, x_{n}$ of points of $X$ an element $\phi\left(x_{0}, \cdots, x_{n}\right) \in J$, such that $\phi\left(g x_{0}, \cdots, g x_{n}\right)$ $=g \cdot \phi\left(x_{0}, \cdots, x_{n}\right)$ for all $g \in G$. The resulting cohomology group we denote by $H^{n}(X / G ; J)$. This notation can be motivated by showing that it is a cohomology group of $X / G$ with coefficients in a certain sheaf each stalk of which is $J$.

Fix now

$$
W: 0 \leftarrow L \leftarrow W_{0} \leftarrow W_{1} \leftarrow \cdots \leftarrow W_{n} \leftarrow \cdots,
$$

a free acyclic resolution of the group $G$. Since $W$ is acyclic, for each $n \geqq 0$ there is the exact sequence

$$
0 \rightarrow Z_{n} \rightarrow W_{n} \rightarrow Z_{n-1} \rightarrow 0 \quad\left(Z_{n}=Z_{n}(W)\right),
$$

and the corresponding exact sequence

$$
\cdots \rightarrow H^{n}\left(X / T ; W_{n}\right) \rightarrow H^{n}\left(X / T, Z_{n-1}\right) \stackrel{\delta^{*}}{\rightarrow} H^{n+1}\left(X / T ; Z_{n}\right) \cdots
$$

In the above we interpret $Z_{-1}=L$. In particular there is the sequence of coboundaries

$$
\begin{aligned}
H^{0}(X / T ; L) \stackrel{\delta^{*}}{\rightarrow} H^{1}\left(X / T ; Z_{0}\right) \rightarrow & \cdots \\
& \rightarrow H^{n-1}\left(X / G, Z_{n-2}\right) \stackrel{\delta^{*}}{\rightarrow} H^{n}\left(X / G ; Z_{n-1}\right) .
\end{aligned}
$$

Now $H^{0}(X / T ; L)$ is the ordinary cohomology group; let $\epsilon$ denote its unit class. Let $c^{n}=c^{n}(X ; L)$ denote the image of $\epsilon$ under the above composition; note that it is also a function of the particular resolution 
$W$. If $m: X \rightarrow Y$ is equivariant, the induced map $H^{n}\left(Y / T ; Z_{n-1}\right)$ $\rightarrow H^{n}\left(X / T ; Z_{n-1}\right)$ maps $c^{n}(Y ; L)$ into $c^{n}(X ; L)$.

If now $W^{\prime}$ is another free acyclic resolution of $G$, there is an equivariant chain map $\psi: W \rightarrow W^{\prime}[5]$; in particular there is the homomorphism $Z_{n-1} \rightarrow Z_{n-1}^{\prime}$ of cycle groups. The induced homomorphism $H^{n}\left(X / G ; Z_{n-1}\right) \rightarrow H^{n}\left(X / G ; Z_{n-1}^{\prime}\right)$ maps the class $c^{n}$ defined by means of $W$ into the class $c^{\prime n}$ defined by means of $W^{\prime}$. Hence $c^{n}=0$ if and only if $c^{\prime n}=0$, since there is also an equivariant chain map $W^{\prime} \rightarrow W$.

We seek now the generalization of the equation $c^{m} \cdot c^{n}=c^{m+n}$ for the mod 2 classes of involutions. In so doing we need a homomorphism $Z_{m-1} \otimes Z_{n-1} \rightarrow Z_{m+n-1}$. We may get a family of such by considering the join $W \circ W$ given by

$$
(W \circ W)_{n}=\sum_{p+q=n-1} W_{p} \otimes W_{q}
$$

where $W_{-1}=L$. Then $W \circ W$, with its customary boundary, is another free acyclic resolution of $G$. Moreover, $Z_{m-1}(W) \otimes Z_{n-1}(W)$ $\subset Z_{m+n-1}(W \circ W)$. There exist, now, equivariant chain maps $W \circ W$ $\rightarrow W$, inducing $Z_{m+n-1}(W \circ W) \rightarrow Z_{m+n-1}(W)$. For each such choice, we get a homomorphism $Z_{m-1} \otimes Z_{n-1} \rightarrow Z_{m+n-1}$, and also

$$
H^{m}\left(X / G ; Z_{m-1}\right) \otimes H^{n}\left(X / G ; Z_{n-1}\right) \rightarrow H^{m+n}\left(X / G ; Z_{m+n-1}\right) .
$$

We now state without proof the following fact.

(4.3) The above homomorphism maps $c^{m}(X ; L) \otimes c^{n}(X ; L)$ into $c^{m+n}(X ; L)$.

Return now to involutions without fixed points. That is, in the above let $G=Z_{2}$. There is the resolution $W$ with each $W_{i}$ the group ring $L\left(Z_{2}\right)$ with elements $l+l_{2} T$ and $\partial: W_{n} \rightarrow W_{n-1}$ multiplication by $1+T$ or $1-T$ according as $n$ is even or odd. There are now only two sequences (4.1). These can be seen to give rise to the two Smith sequences for the involution $T$ without fixed points [15]. Of course if the coefficient ring is in fact $L=Z_{2}$, then $Z_{n-1}(W)=Z_{2}$ for all $n$. Then

$$
c^{m} \in H^{m}\left(X / T ; Z_{m-1}(W)\right)=H^{m}\left(X / T ; Z_{2}\right)
$$

is the classical $m$ th power of the fundamental class, already referred to.

We are now in a position to define a homological co-index. Ours is a generalized version of Yang's index [18].

(4.4) Definition. If a finite group $G$ operates freely on a paracompact space $X$, and if $L$ is a principal ideal domain, define co-ind ${ }_{L} X$ to be the largest integer $n$ for which the class $c^{n}(X ; L)$ is not zero. We write co-ind ${ }_{L} X=-1$ if and only if $X=\varnothing$. 
Note that it follows from (4.3) that if $c^{n}=0$ then $c^{m}=0$ for all $m \geqq n$.

For consistency we shall now return to fixed point free involutions. (4.5) We have

$$
\text { ind } X \leqq \operatorname{co-ind~}_{L} X \leqq \operatorname{co-ind~} X
$$

for any coefficient group $L$ which has co-ind $_{L} S^{n}=n$.

This follows from the fact that if there exists an equivariant map $X \rightarrow Y$ then co-ind ${ }_{L} Y \geqq$ co-ind $_{L} X$. We come now to a result, suggested by a theorem of Eilenberg (Duke Math. J. vol. 6 (1940) pp. 428-437), very reminiscent of the result for index in (3.10).

(4.6) Theorem. Suppose that $T$ and $T^{\prime}$ are fixed point free involutions on paracompact spaces $X$ and $Y$ respectively, and that co-ind ${ }_{L} X$ $=$ co-ind $_{L} Y=n<\infty$. If $m: X \rightarrow Y$ is equivariant, then $m^{*}: H^{n}(Y ; L)$ $\rightarrow H^{n}(X ; L)$ is nontrivial.

For consider a free acyclic resolution $W$ of $Z_{2}$ with each $W_{n}$ finitely generated. The equivariant cohomology group $H^{n}(X / G ; L(G))$ may be naturally identified with $H^{n}(X, L)$. Hence, $H^{n}\left(X / G ; W_{n}\right)$ is isomorphic with a finite number of copies of $H^{n}(X ; L)$. From (4.2) we get a commutative diagram

$$
\begin{gathered}
\sum H^{n}(Y ; L) \approx H^{n}\left(Y / G ; W_{n}\right) \stackrel{\tau^{*}}{\rightarrow} H^{n}\left(Y / G ; Z_{n-1}\right) \stackrel{\delta^{*}}{\rightarrow} H^{n+1}\left(Y / G, Z_{n}\right) \\
\downarrow m_{1}^{*} \\
\sum m_{2}^{*}(X ; L) \approx H^{n}\left(X / G ; W_{n}\right) \rightarrow H^{n}\left(X / G ; Z_{n-1}\right) \stackrel{\delta^{*}}{\rightarrow} H^{n+1}\left(X / G, Z_{n}\right) .
\end{gathered}
$$

The element $c^{n}(Y ; L)$ of $\left.\left.H^{n}\right) Y / G ; Z_{n-1}\right)$ is killed by $\delta^{*}$. Hence there exists a $b \in H^{n}\left(X / G ; W_{n}\right)$ with $\tau^{*}(b)=c^{n}$. Now $m_{2}^{*}$ does not kill $c^{n}$, and hence $m_{1}^{*}$ does not kill $b$. However, if $m^{*}: H^{n}(Y ; L) \rightarrow H^{n}(X ; L)$ were trivial, so also would $m_{1}^{*}$ be trivial. The theorem follows, so does the following corollary.

(4.7) If $T$ is a fixed point free involution on a paracompact space $X$, and if $n=$ co-ind $_{L} X$, then $H^{n}(X ; L) \neq 0$.

It can be seen that co-ind ${ }_{L} X$, for a connected $X$ is $\geqq 1$ if $L$ contains no element $l$ with $2 l=1$. In particular, it follows from (4.7) that co-ind ${ }_{L} S^{n}=n$ for such $L$. In particular, such is the case for $Z_{2}$ and $Z$.

Consider now the case where $L=Z$. Now the class $c^{n+1}(X ; Z)$ is an element of the equivariant cohomology group $H^{n+1}\left(X / T ; Z_{n}(W)\right)$, which for the "standard" resolution of $Z_{2}$ is

$$
H^{n+1}\left(X / T ; H_{n}\left(S^{n}\right)\right) \approx H^{n+1}\left(X / T ; \pi_{n}\left(S^{n}\right)\right) .
$$

This may be considered as the cohomology of $X / T$ with coefficients in a certain sheaf with stalks $\pi_{n}\left(S^{n}\right)$. On the other hand, we have con- 
sidered already in $\$ 3$ the primary obstruction to an equivariant mapping. The primary obstruction to an equivariant mapping $X \rightarrow S^{n}$ is an element of $H^{n+1}\left(X / T ; B\left(\pi_{n}\left(S^{n}\right)\right)\right)$, for $X$ cellular. Now for $X$ and $T$ cellular, these coincide, so that we may consider $c^{n+1}(X ; Z)$ as the primary obstruction to an equivariant mapping $X \rightarrow S^{n}$.

We can now finish the proof of (3.12) for the cases $n=1,2$. In particular, suppose for example that $X$ is a finite complex, that $T: X \rightarrow X$ is cellular, and that co-ind $X=2$. We show that co-ind $z$ $=2$. If $\operatorname{co-ind}_{Z} X \leqq 1$, then the 2 -skeleton $X^{(2)}$ of $X$ would have co-ind $_{z} X^{(2)}=1$. It follows from the above paragraph that there is then an equivariant map $X^{(2)} \rightarrow S^{1}$. But there are then no obstructions to extending this to an equivariant map $X \rightarrow S^{1}$. Hence co-ind $z X=2$. But then every equivariant $F: X \rightarrow S^{2}$ has $f^{*}: H^{2}\left(S^{2}\right) \rightarrow H^{2}(X)$ nontrivial by (4.6). Then $f$ is essential.

Note also that it follows gracefully from (4.6) that co-ind $P_{x_{0}}\left(S^{n}\right)$ $=n$ for $n$ odd and $\geqq 3$. For by (4.7), co-ind $S_{L}=n-1$. If also co-ind $P_{x_{0}}\left(S^{n}\right)=n-1$, then every equivariant $f: P_{x_{0}}\left(S^{n}\right) \rightarrow S^{n-1}$ would have $f^{*}: H^{n-1}\left(S^{n-1} ; Z\right) \rightarrow H^{n-1}\left(P_{x_{0}}\left(S^{n}\right) ; Z\right)$ nontrivial by (4.6). But every nonzero element of $H^{n-1}\left(P_{x_{0}}\left(S^{n}\right) ; Z\right)$ has a nonzero square, so we get a contradiction.

We shall next examine common features of the various co-index functions. In so doing, it is convenient to introduce an abstract coindex. Say that a collection $J$ of fixed point free involutions is hereditary if and only if

(a) whenever $T: X \rightarrow X$ is in $J$ and $A$ is a closed invariant subset of $X$ then the restriction $T: A \rightarrow A$ is in $J$;

(b) the antipodal map $A$ on $S^{0}$ is in $J$.

(4.8) Definition. A Co-index function on a hereditary collection of fixed point free involutions assigns to each $(T, X)$ in $J$ with $X \neq \varnothing$ a non-negative integer or $\infty$, and satisfies:

(i) if $m: X \rightarrow Y$ is an equivariant map between two involutions of $J$ then Co-ind $X \leqq$ Co-ind $Y$;

(ii) if $X=A \cup B$ where $A$ and $B$ are closed invariant sets in $X$ and $(T, X) \in J$, then

$$
\text { Co-ind } X \leqq \text { Co-ind } A+\text { Co-ind } B+1 \text {; }
$$

(iii) Co-ind $S^{0}=0$.

We can now characterize co-index among all the Co-index functions.

(4.9) The co-index function is a Co-index function on the class of fixed point free involutions on normal spaces. For any hereditary collection $J$ of involutions $T$ on normal spaces $X$, and any Co-index function on $J$, 


$$
\text { Co-ind } X \leqq \operatorname{co-ind} X, \quad \operatorname{all}(T, X) \in J \text {. }
$$

The first part of (4.9) follows easily from (3.4). In proving the second part, we may assume co-ind $X<\infty$. We proceed by induction on co-ind $X$, assuming that Co-ind $X \leqq$ co-ind $X$ if co-ind $X \leqq n-1$. Now let co-ind $X=n$. By (3.4), express $X$ as the union of $n+1$ closed invariant subsets $A_{1}, \cdots, A_{n+1}$ each of which admits a cross-section. Let $A=A_{1} \cup \cdots \cup A_{n}, B=A_{n+1}$. Then

$$
\text { Co-ind } X \leqq \text { Co-ind } A+\text { Co-ind } B+1 \text {. }
$$

Since $B$ admits a cross-section there is an equivariant map $B \rightarrow S^{0}$; hence

$$
0 \leqq \text { Co-ind } B \leqq \text { Co-ind } S^{0}=0 .
$$

Since Co-ind $A \leqq n-1$ by the induction, we have Co-ind $X \leqq n$. Hence (4.9) follows.

Our next point is that co-ind ${ }_{L}$ is a Co-index function on the class of fixed point free involutions on paracompact spaces. The only comment needed concerns

$$
\operatorname{co-ind}_{L} X \leqq \operatorname{co-ind}_{L} A+\operatorname{co-ind}_{L} B+1,
$$

where $X$ is the union of closed invariant sets $A$ and $B$. Let $n_{1}$ $=\operatorname{co-ind}_{L} A$ and $n_{2}=\operatorname{co-ind}_{L} B$. Then

$$
H^{n_{1}+1}\left(X / T ; Z_{n_{1}}\right) \rightarrow H^{n_{1}+1}\left(A / T ; Z_{n_{1}}\right)
$$

kills $c^{n_{1}+1}(X ; L)$; similarly for $c^{n_{2}+1}(X ; L)$. Since $X / T=A / T \cup B / T$ it follows from a well known principle of products, and the relation $c^{n_{1}+1}(X ; L) \otimes c^{n_{2}+1}(X ; L) \rightarrow c^{n_{1}+n_{2}+2}(X ; L)$ of $(4.3)$, that $c^{n_{1}+n_{2}+2}=0$. Hence co-ind $\mathrm{i}_{L} X \leqq n_{1}+n_{2}+1$, and

(4.10) The function co-ind ${ }_{L} X$ is a Co-index function.

Both co-ind and co-ind ${ }_{L}$ share the following property, which we call the continuity property:

Say that Co-ind is continuous on $J$ if whenever $(T, X) \in J$ and $A$ is a closed invariant subset of $X$, then there exists a closed invariant neighborhood $C$ of $A$ with Co-ind $A=$ Co-ind $C$.

(4.11) Both co-ind and co-ind $d_{L}$ are continuous on the class of fixed point free involutions on paracompact spaces.

Note first that if $A$ and $C$ are invariant and $A \subset C$, then the inclusion map is equivariant so that $\mathrm{Co}$-ind $A \leqq \mathrm{Co}$-ind $C$. We prove the opposite inequalities. We may as well assume co-ind $A$ [or co-ind ${ }_{L} A$ ] finite. The inequality for co-ind ${ }_{L}$ is an application of the continuity of AWS-cohomology. Suppose now co-ind $X<\infty$. Cover $A$ by closed invariant sets $A_{1}, \cdots, A_{n+1}$ each admitting a cross-section. Write 
$A_{i}=B_{i} \cup T\left(B_{i}\right), B_{i}$ closed and $B_{i} \cap T\left(B_{i}\right)=\varnothing$. By normality there are open sets $U_{i} \supset B_{i}$ such that $U_{i} \cap T\left(U_{i}\right)=\varnothing$. Let $U=\cup U_{i} \cup \cup T\left(U_{i}\right)$. Any closed neighborhood $C$ of $A$ with $A \subset C \subset U$ then satisfies co-ind $C$ $\leqq$ co-ind $A$.

We can now give Yang's generalization of the Borsuk-Ulam mapping theorem [18].

(4.12) Let $T$ be a fixed point free involution on a paracompact space $X$, and let $f: X \rightarrow R^{r}$ be any map of $X$ into Euclidean $r$-space. Let $A \subset X$ denote all $x \in X$ with $f(x)=f(T x)$. Then $A$ is a closed invariant subset of $X$, and

$$
\text { Co-ind } A \geqq \text { Co-ind } X-r
$$

for any continuous Co-index with Co-ind $S^{n}=n$, all $n$.

In particular, $A$ is nonempty for all $f$ if and only if co-ind $X \geqq r$.

We have seen that appropriate coefficient groups for an involution are $Z$ and $Z_{2}$. It is not surprising that $Z_{2}$, being a field, should yield more striking general properties than does $Z$. For example, denoting co-ind $z_{2}$ by co-ind $d_{2}$, we have the following theorem of Yang [17]: if $X_{1}$ and $X_{2}$ are closed invariant subsets of $S^{n}$, then

$$
\text { co-ind }_{2} X_{1}+\text { co-ind }_{2} X_{2}-\text { co-ind }_{2} X_{1} \cap X_{2} \leqq n \text {. }
$$

It can be seen that this is actually equivalent to the following duality result: if $X$ is a closed invariant subset of $S^{n}$ and if $V$ is an invariant neighborhood of $X$ with co-ind ${ }_{2} \bar{V}=\operatorname{co-ind}_{2} X=r$, then

$$
\text { co-ind }_{2}\left(S^{n}-V\right)=n-r-1 .
$$

These results are no doubt false for $Z$. One could hazard a guess at the distinction by saying that there should be in some sense a function $\operatorname{ind}_{L} X$, that always

$$
\operatorname{co-ind}_{L}\left(S^{n}-V\right)=n-\operatorname{ind}_{L} X-1,
$$

but that for $L=Z_{2}$ we actually have $\operatorname{ind}_{2} X=$ co-ind $_{2} X$, all $X$.

5. The suspension of an involution. If $T$ is a fixed point free involution on a space $X$, there is the suspended involution $T$ on the suspension $S(X)$ of $X$ (see $\S 2)$. There is also the equivariant embedding $X \subset S(X)$. It is easily seen that

$$
\begin{aligned}
\text { ind } S(X) & \geqq \text { ind } X+1, \\
\text { co-ind } S(X) & \leqq \text { co-ind } X+1 .
\end{aligned}
$$

Unfortunately the inequalities in the above can occur. 
(5.1) Let $T$ be a fixed point free involution on a compact space $X$. Then

$$
\operatorname{co-ind}_{L} S(X)=\operatorname{co-ind}_{L} X+1
$$

for $L=Z, Z_{2}$.

For, in the first place, it is true for every $L$ that

$$
\operatorname{co-ind}_{L} S(X) \leqq \operatorname{co-ind}_{L} X+1 \text {. }
$$

For there is an invariant neighborhood $V$ of $X$ in $S(X)$ with co-ind ${ }_{L} \bar{V}$ $=$ co-ind $_{L} X$. Moreover, $S(X)-V$ admits a cross-section, so that co-ind $_{L}(S(X)-V)=0$. Then

$$
\begin{aligned}
\operatorname{co-ind}_{L} S(X) & \leqq \operatorname{co-ind}_{L} V+\operatorname{co-ind}_{L}(S(X)-V)+1 \\
& \leqq \operatorname{co-ind}_{L} X+1
\end{aligned}
$$

Suppose now that $n=$ co-ind $_{L} S(X)=$ co-ind $_{L} X<\infty$. By (4.6), the equivariant inclusion $i: X \rightarrow S(X)$ induces a nontrivial $i^{*}: H^{n}(S(X) ; L)$ $\rightarrow H^{n}(X ; L)$. But $X$ is contractible to a point in $S(X)$, so that $n=0$. However, always $1 \leqq$ ind $S(X) \leqq$ co-ind $_{L} S(X)$ for $L=Z_{2}, Z$. Thus we see

$$
\operatorname{co-ind}_{L} S(X)=\operatorname{co-ind}_{L} X+1 \text {. }
$$

It is reasonable to summarize the above by saying that co-ind $z$ and co-ind $z_{2}$ are stable under suspension.

Let $\left(T, S^{k}(X)\right)$ denote the $k$-fold suspension of $X$ with its suspended involution $T$.

(5.2) Definition. If $T$ is a fixed point free involution on a space $X$, then ind $X$ [co-ind $X]$ is stable if and only if for every $k \geqq 0$, ind $S^{k}(X)=$ ind $X+k$ [co-ind $S^{k}(X)=$ co-ind $X+k$ ].

It follows from our preceding results that there are several cases in which index and co-index are stable.

(5.3) Suppose that $T$ is a fixed point free involution on a compact $(n-1)$-connected space, $n \geqq 2$. If ind $X \leqq 2 n-2$, then ind $X$ is stable.

Suppose by way of induction that we have shown ind $S^{k}(X)$ $=$ ind $X+k$. Consider now $S^{k+1}(X)$. The suspension $S^{k}(X)$ is $(n+k-1)$-connected, and ind $S^{k}(X) \leqq 2 n+k-2$. We wish to prove that ind $S^{k+1}(X)<2 n+k$. Consider then the (empty) set $E_{*}\left(S^{2 n+k-1}, S^{k}(X)\right)$ of equivariant homotopy classes. Theorem 2.5 shows that $s_{*}$ maps $E_{*}\left(S^{2 n+k-1}, S^{k}(X)\right)$ onto $E_{*}\left(S^{2 n+k}, S^{k+1}(X)\right)$. Hence $E_{*}\left(S^{2 n+k}, S^{k+1}(X)\right)=\varnothing$, and ind $S^{k+1}(X) \leqq 2 n+k-1$. Similar use of (2.5) proves the following.

(5.4) Let $A$ be a simplicial fixed point free involution on a finite 
simplicial complex. If $\operatorname{dim} K \leqq 2$ co-ind $K-3$, then co-ind $K$ is stable. Let us note finally one more case in which stability holds. If ind $X=\operatorname{co-ind}_{L} X\left(L=Z_{2}\right.$ or $\left.Z\right)$ then ind $X$ is stable. If $\operatorname{co-ind}_{L} X$ $=$ co-ind $X$, then co-ind $X$ is stable.

The space $P_{x_{0}}\left(S^{n}\right), n$ odd and $\geqq 3$, furnishes an example of a space for which co-index is unstable. We shall now briefly sketch an example which is a finite simplicial complex. It is natural to seek such an example in $P_{x_{0}}\left(S^{n}\right)$. So, having fixed $x_{0} \in S^{n}$, let $V \subset S^{n} \times S^{n} \times S^{n}$ consist of all triples $\left(x_{1}, x_{2}, x_{3}\right)$ such that in

$$
x_{0}, x_{1}, x_{2}, x_{3}, A\left(x_{0}\right),
$$

no adjacent pair is antipodal. $V$ is then an open subset of $S^{n} \times S^{n} \times S^{n}$. There is the involution $T: S^{n} \times S^{n} \times S^{n} \rightarrow S^{n} \times S^{n} \times S^{n}$ given by $T\left(x_{1}, x_{2}, x_{3}\right)=\left(A\left(x_{3}\right), A\left(x_{2}\right), A\left(x_{1}\right)\right) ; V$ is invariant under $T$. Now we may embed $V$ equivariantly in $P_{x_{0}}\left(S^{n}\right)$ in a standard fashion. Namely, assign to $\left(x_{1}, x_{2}, x_{3}\right)$ the path from $x_{0}$ to $A\left(x_{0}\right)$ which follows the unique shortest geodesic from $x_{0}$ to $x_{1}$, then the shortest geodesic from $x_{1}$ to $x_{2}$, then from $x_{2}$ to $x_{3}$, and finally from $x_{3}$ to $A\left(x_{0}\right)$. By a direct computation using Morse's methods it can be seen that $j^{*}: H^{i}\left(P_{x_{0}} ; Z\right)$ $\approx H^{i}(V ; Z)$ for $i \leqq 3 n-3$. Now we can show that, since $V$ has the cohomology of $P_{x_{0}}\left(S^{n}\right)$ in dimensions $\leqq 2 n-2$, co-ind $V=n$, just as was done for $P_{x_{0}}\left(S^{n}\right)$. However, we wish to obtain an example of a finite simplicial complex so we seek further refinements.

Let $B=S^{n} \times S^{n} \times S^{n}-V$. Now $B$ is a finite subcomplex of $S^{n} \times S^{n}$ $X S^{n}$, invariant under the involution $T$. Instead of removing $B$ from $S^{n} \times S^{n} \times S^{n}$, remove instead the second regular neighborhood $N^{2}(B)$. Let $K=S^{n} \times S^{n} \times S^{n}-N^{2}(B)$. Now $K$ is a deformation retract of $V$. Hence with the above identification of $V$ as a subset of $P_{x_{0}}\left(S^{n}\right)$, we have $i^{*}: H^{i}\left(P_{x_{0}} ; Z\right) \approx H^{i}(K ; Z)$ for $i \leqq 3 n-3$. Moreover, $K \subset V$ is invariant under $T$; considered as embedded in $P_{x_{0}}\left(S^{n}\right), K$ is then invariant under $\Gamma$. Consider finally the $(2 n-2)$-skeleton $K^{\prime}=K^{(2 n-2)}$ of $K$. Then $K^{\prime}$ is invariant under $\Gamma$. Moreover, $j^{*}: H^{i}\left(P_{x_{0}} ; Z\right)$ $\rightarrow H^{i}\left(K^{\prime} ; Z\right)$ is an isomorphism for $i<2 n-2$ and a monomorphism for $i=2 n-2$. In particular every nonzero element of $H^{n-1}\left(K^{\prime} ; Z\right)$ has a nonzero square in $H^{2 n-2}\left(K^{\prime} ; Z\right)$ for $n$ odd. That is, we can prove that co-ind $K^{\prime}>n-1$, for $n$ odd and $\geqq 3$, just as was done for $P_{x_{0}}\left(S^{n}\right)$. Since $K^{\prime} \subset P_{x_{0}}\left(S^{n}\right)$, it follows that co-ind $K^{\prime}=n$, and that there exists an inessential equivariant map $K^{\prime} \rightarrow S^{n}$ (this is the map $x_{1}, x_{2}, x_{3} \rightarrow x_{2}$ ). Hence also co-ind $S\left(K^{\prime}\right)=n$, and co-ind $K^{\prime}$ is unstable. This is also the example mentioned in the introduction. In particular, it is of the lowest possible dimension in order to possess inessential equivariant maps into $S^{n}$ and also have co-index $n$. 
A direction is now clear; namely stabilize index and co-index.

(5.5) Definition. Let $T$ be a fixed point free involution on a compact space $X$. Define the stable index and stable co-index by

$$
\begin{aligned}
\operatorname{ind}_{s} X & =\text { l.u.b. }\left\{\text { ind } S^{k}(X)-k: k \geqq 0\right\}, \\
\text { co-ind }_{s} X & =\text { g.l.b. }\left\{\operatorname{co-ind} S^{k}(X)-k ; k \geqq 0\right\} .
\end{aligned}
$$

We have immediately that

$$
\text { ind } \begin{aligned}
X & \leqq \operatorname{ind}_{s} X \leqq \operatorname{co-ind}_{L} X \leqq \operatorname{co-ind}_{s} X \\
& \leqq \text { co-ind } X \text { for either } L=Z \text { or } Z_{2} .
\end{aligned}
$$

Following Spanier's notation [12] let $\sum_{n}(X)$ denote the stable homotopy group of $X$ and $\sum^{n}(X)$ the stable cohomotopy group. The following should be compared with (3.11), (3.12), and (4.7).

(5.6) Let $T$ be a fixed point free involution on a compact space $X$. If ind $_{s} X=n$ then $\sum_{n}(X) \neq 0$. If co-ind ${ }_{s} X=m$ then $\sum^{m}(X) \neq 0$.

An equivariant suspension category for fixed point free involutions and equivariant maps exactly analogous to the Spanier-Whitehead $S$-category can be introduced [12]. An equivariant duality theorem can be proved by methods entirely analogous to those in [12].

Let $K \subset S^{n}$ be an invariant subcomplex of $\left(A, S^{n}\right)$ in some fixed triangulation of $S^{n}$. An equivariant $n$-dual $D_{n}(K)$ is an invariant subcomplex of $S^{n}-K$ which is an equivariant deformation retract of $S^{n}-K$.

(5.7) With notation as above, we have

$$
\begin{aligned}
\operatorname{co-ind}_{s} D_{n}(K) & =n-\operatorname{ind}_{s} K-1, \\
\operatorname{ind}_{s} D_{n}(K) & =n-\operatorname{co-ind}_{s} K-1 .
\end{aligned}
$$

It is easily seen that the complement of the second regular neighborhood of $K$ in $S^{n}$ may be taken as a $D_{n}(K)$ [12]. The above property shows a close correspondence between the stable index and coindex, and the homological co-index of $\S 4$.

Of necessity, this section contains only brief statements and sketches. For one thing, it is not at all clear what the future holds for stable index and co-index. But it is plausible that the problem of computing and studying these stabilized functions is easier than the original questions (1) and (2) pointed out in the introduction.

6. The involution of a sphere bundle. The primary example of a fixed point free involution being the antipodal map of a sphere, it is natural to consider in some detail the antipodal map of a bundle of 
spheres. We consider then in this section an $n$-sphere bundle $p: B \rightarrow X$, where $X$ is paracompact and the structural group is $0(n+1)$.

Always in a fibre bundle the center of the structural group acts as a transformation group on the bundle space. The center of $0(n+1)$ containing as its nontrivial element the antipodal map of $S^{n}$, we obtain a fixed point free involution $T: B \rightarrow B$, the antipodal map on each fibre $S^{n}=B_{x}$. Denote the orbit space $B / T$ of $(T, B)$ by $B^{*}$. There is the natural map $q: B^{*} \rightarrow X$, a bundle of projective $n$-spaces $P_{n}$. Note that the concept of a 0 -sphere bundle coincides with the concept of a fixed point free involution. In particular if $p: B \rightarrow X$ is a 0 -sphere bundle then $q: B^{*} \rightarrow X$ is a homeomorphism; in this case we identify $B^{*}$ and $X$.

Since we prefer to use sphere bundles instead of vector space bundles, the concept of Whitney join replaces that of Whitney sum. We use the following notation. If $p_{i}: B_{2} \rightarrow X, i=1,2$, are sphere bundles, there is the sphere bundle $p_{1} \circ p_{2}: B_{1} \circ B_{2} \rightarrow X$ whose fibres $\left(B_{1} \circ B_{2}\right)_{x}$ are the ordinary joins $B_{1 x} \circ B_{2 x}$ of the fibres of $B_{1}$ and $B_{2}$. Denote a typical element of $\left(B_{1} \circ B_{2}\right)_{x}$ by $(1-t) b_{1}+t b_{2}$ where $0 \leqq t \leqq 1, b_{i} \in B_{i x}$. The involution $T: B_{1} \circ B_{2} \rightarrow B_{1} \circ B_{2}$ has $B_{1}$ and $B_{2}$ as invariant closed subspaces; the orbit space $\left(B_{1} \circ B_{2}\right)^{*}$ has $B_{1}^{*}$ and $B_{2}^{*}$ as subspaces.

Let us apologize in advance for giving an outline of Whitney classes; the reader will see that our treatment is inspired by those of Borel [1] and Hirzebruch [8]. However, since our primary interest is in involutions, it is necessary that we define the Whitney classes in terms of the involution $T$.

For a sphere bundle $p: B \rightarrow X$, the additive cohomology of $B^{*}$ is completely determined; here and throughout the section it is to be assumed that all cohomology has coefficients $Z_{2}$. The fibre $P_{n}$ of $q: B^{*} \rightarrow X$ is totally nonhomologous to 0 in $B^{*}$. For the classes $c^{i} \in H^{i}\left(P_{n}\right)$ of $\left(T, S^{n}\right)$ are the images of the classes $c^{i} \in H^{i}\left(B^{*}\right)$ of $(T, B)$. In particular, then, $q^{*}: H^{k}(X) \rightarrow H^{k}\left(B^{*}\right)$ is a monomorphism. Also every $\beta_{k} \in H^{k}\left(B^{*}\right)$ can be uniquely expressed as

$$
\beta_{k}=q^{*} \alpha_{k}+q^{*} \alpha_{k-1} \cdot c+\cdots+q^{*} \alpha_{k-n} \cdot c^{n}
$$

where $\alpha_{i} \in H^{i}(X)$ and $c$ is the fundamental class of $(T, B)$.

Having obtained the additive structure of $H\left(B^{*}\right)$, consider now the product structure and in particular the powers $c^{i}, i>n$. We have from (6.1) that

$$
c^{n+1}=q^{*} w_{n+1}+q^{*} w_{n} \cdot c+\cdots+q^{*} w_{1} \cdot c^{n}
$$

for unique classes $w_{i} \in H^{i}(X), i=1, \cdots, n+1$. For a given space $Y$, denote by $H^{*}(Y)$ the strong direct sum $\sum_{0}^{\infty} H^{i}(Y)$; consider an ele- 
ment of $H^{*}(Y)$ as a formal power series $\sum \alpha_{i} t^{i}, \alpha_{i} \in H^{i}(Y)$. In particular, let $w=w(B)$ denote $\sum_{0}^{n+1} w_{i} t^{i} \in H^{*}(X)$ for a sphere bundle $p: B \rightarrow X$, where $w_{0}=1$.

(6.3) The function assigning to each sphere bundle $p: B \rightarrow X$ the class wE $H^{*}(X)$ is characterized by the following properties:

(a) for a 0 -sphere bundle, $w=1+c t$ where $c$ is the fundamental class of $(T, B)$;

(b) if $p_{i}: B_{i} \rightarrow X_{i}$ are sphere bundles and $f: B_{1} \rightarrow B_{2}$ is a bundle map, then the induced map $\bar{f}: X_{1} \rightarrow X_{2}$ has $\bar{f}^{*} w\left(B_{2}\right)=w\left(B_{1}\right)$;

(c) if $p_{i}: B_{i} \rightarrow X, i=1,2$, are sphere bundles then $w\left(B_{1} \circ B_{2}\right)$ $=w\left(B_{1}\right) \cdot w\left(B_{2}\right)$.

We shall prove that (c) holds. In $B_{1} \circ B_{2}$, let $C_{1}$ denote all points of fibres $\left(B_{1} \circ B_{2}\right)_{x}$ of the form $(1-t) b_{1}+t b_{2}$ with $t \leqq 1 / 2$, and let $C_{2}$ be all such with $t \geqq 1 / 2$. Then $B_{1} \circ B_{2}=C_{1} \cup C_{2}, C_{i} \supset B_{i}$ and $B_{i}$ is an equivariant deformation retract of $C_{i}$. Passing to orbit spaces, $\left(B_{1} \circ B_{2}\right)^{*}=C_{1}^{*} \cup C_{2}^{*}, C_{i}^{*} \supset B_{i}^{*}$ and $B_{i}^{*}$ is a deformation retract of $C_{i}^{*}$. Hence $H^{k}\left(C_{i}^{*}\right) \approx H^{k}\left(B_{i}^{*}\right)$. Also if $\beta_{i} \in H\left(\left(B_{1} \circ B_{2}\right)^{*}\right)$ and $H\left(\left(B_{1} \circ B_{2}\right)^{*}\right)$ $\rightarrow H\left(B_{i}^{*}\right)$ kills $\beta_{i}, i=1,2$, then $\beta_{1} \cdot \beta_{2}=0$.

Suppose then that $B_{1}$ is an $m$-sphere bundle and $B_{2}$ an $n$-sphere bundle. Then by (6.2)

$$
c^{m+1}+q^{*} w_{1}\left(B_{1}\right) \cdot c^{m}+\cdots+q^{*} w_{m+1}\left(B_{1}\right) \in H^{m+1}\left(\left(B_{1} \circ B_{2}\right)^{*}\right)
$$

is killed under restriction to $B_{1}^{*}$, and

$$
c^{m+1}+q^{*} w_{i}\left(B_{2}\right) \cdot c^{n}+\cdots+q^{*} w_{n+1}\left(B_{2}\right) \in H^{n+1}\left(\left(B_{1} \circ B_{2}\right)^{*}\right)
$$

is killed by restriction to $B_{2}^{*}$. Hence their product is 0 in $\left(B_{1} \circ B_{2}\right)^{*}$. If one computes the product and compares with

$$
c^{m+n+2}=\sum_{1}^{m+n+2} q^{*} w_{i}\left(B_{1} \circ B_{2}\right) \cdot c^{m+n+2-i}
$$

from (6.2), one obtains the result.

Since the Whitney classes $w_{i}$ of a bundle also satisfy (a), (b), and (c) we see that our classes $w_{i}$ are the Whitney classes.

We need the notation of the proof of uniqueness. One considers three sphere bundles over $B^{*}$. There is the 0 -sphere bundle $\nu: B \rightarrow B^{*}$. Thinking of $B^{*}$ as the collection of all antipodal pairs of $B$, there is the induced bundle $B_{1} \rightarrow B^{*}$, which assigns to each element $b^{*}$ (an antipodal pair) of $B^{*}$ the fibre of $B$ which contains $b^{*}$. There is also the sphere bundle $B_{2} \rightarrow B^{*}$ assigning as fibre to each $b^{*}$ the orthogonal $(n-1)$-sphere to $b^{*}$ in the $n$-sphere $B_{x}$ which contains $b^{*}$. Now, as sphere bundles over $B^{*}, B_{1}=B \circ B_{2}$. The class of $B_{1} \rightarrow B^{*}$ is $q^{*} w$, 
where $w$ is the original class of $p: B \rightarrow X$, and the class of the 0 -sphere bundle $B \rightarrow B^{*}$ is $1+c t$. Hence by (c),

$$
\begin{aligned}
1+q^{*} w_{1} \cdot t+\cdots+q^{*} w_{n+1} \cdot t^{n+1} & \\
& =(1+c t)\left(1+\alpha_{1} t+\cdots+\alpha_{n} t^{n}\right)
\end{aligned}
$$

where $\sum \alpha_{i} i^{i}$ is the class of $B_{2} \rightarrow B^{*}$.

In the ring $H^{*}(X)$, w has a unique inverse $\bar{w}=\sum \bar{w}_{i} t^{i}$. In $H^{*}\left(B^{*}\right)$, $1+c t$ has inverse $1+c t+c^{2} t^{2}+\cdots$. Hence we get

$$
\begin{aligned}
(6.5)^{\prime} \quad 1+c t+c^{2} t^{2}+\cdots= & \left(1+\alpha_{1} t+\cdots+\alpha_{n} t^{n}\right) \\
& \cdot\left(1+q^{*} \bar{w}_{1} \cdot t+q^{*} \bar{w}_{2} \cdot t^{2}+\cdots\right) .
\end{aligned}
$$

We shall use co-ind ${ }_{2} B$ to denote the $\bmod 2$ co-index of $T: B \rightarrow B$, as in $\S 4$.

(6.6) Theorem. For every $n$-sphere bundle $p: B \rightarrow X$, it is true that

$$
\text { co-ind }_{2} B=n+k \text {, }
$$

where $k$ is the largest integer with the dual Whitney class $\bar{w}_{k}$ nonzero.

Proof. Observe that co-ind ${ }_{2} B$ is the degree of the polynomial $1+c t+c^{2} t^{2}+\cdots$, while $k$ is the degree of $\bar{w}$. Hence it follows from $(6.5)^{\prime}$ that

Now from (6.5),

$$
\text { co-ind }_{2} B \leqq n+k \text {. }
$$

$$
\alpha_{n}=c^{n}+\text { terms of lower degree in } c
$$

while $\alpha_{1}, \cdots, \alpha_{n-1}$ all consist of terms of degree $<n$ in $c$. Hence from $(6.5)^{\prime}$

$$
c^{m}=q^{*} \tilde{w}_{m-n} \cdot c^{n}+\text { terms of lower degree in } c .
$$

Hence by (6.1), $\bar{w}_{m-n} \neq 0$ implies $c_{m} \neq 0$. That is, co-ind $2 B=n+k$.

The following corollary, of a type due originally to Thom [15], suggests the convenience of considering the involution of a sphere bundle.

(6.7) Corollary. If a differentiable $n$-manifold $X$ can be immersed in a differentiable m-manifold $Y$ then

$$
n+k \leqq m+l
$$

where $k, l$ are the largest integers with the dual Stiefel-Whitney classes $\bar{w}_{k}(X), \bar{w}_{l}(Y)$ nonzero. 
For if $X$ can be immersed in $Y$, there is an equivariant map of the bundle $V_{1}(X)$ of unit tangent vectors to $X$ into the bundle $V_{2}(Y)$ of unit tangent vectors to $Y$. Then co-ind $2 V_{1}(X) \leqq$ co-ind $V_{2} V_{1}(Y)$ by (4.5).

We see by (6.6) that if $p: B \rightarrow X$ is an $n$-sphere bundle, it might not be possible to map $B$ equivariantly into, say, $S^{n}$. We shall consider an equivariant map $f$ of $B$ into $R^{m}, m \geqq n+1$, and try to measure the set $f^{-1}(0)$. Note that $B-f^{-1}(0)$ is mapped equivariantly into $S^{m-1}$ and hence has co-ind $\mathrm{d}_{2} \leqq m-1$. We are particularly interested in seeing how many fibres $f^{-1}(0)$ must intersect.

(6.8) Definition. If $A$ is a closed subset of the compact subset of the compact space $X$ and if $\gamma \in H^{n}(X)$, then we say that $A$ is a support of $\gamma$ if for every neighborhood $U$ of $A$, the natural map $H_{c}^{n}(U)$ $\rightarrow H^{n}(X)$ has $\gamma$ in its image. Similarly in Cech homology, $A$ is a support of $c \in H_{n}(X)$ if $c$ is in the image of $H_{n}(A) \rightarrow H_{n}(X)$.

In compact $n$-manifold $X, A$ supports $\gamma \in H^{k}(X)$ iff $A$ supports the dual class $c \in H_{n-k}(X)$ of $\gamma$.

(6.9) Theorem. Suppose that $p: B \rightarrow X$ is an $n$-sphere bundle over the compact space $X$ and that $A$ is a closed subset of $B$, invariant under the antipodal map $T: B \rightarrow B$. If co-ind $_{2}(X-A) \leqq m-1$, then $p(A) \subset X$ is a support for every dual Whitney class $\bar{w}_{p}, p \geqq m-n$.

Proof. Suppose that $U$ is a neighborhood of $p(A)$. Let $V=p^{-1}(U)$; then $V \supset A$. Hence co-ind $2(B-V) \leqq m-1$, and $c^{m}=0$ in $H^{m}\left(B^{*}-V^{*}\right)$. But in $B^{*}$,

$$
c^{m}=q^{*} \bar{w}_{m-n} \cdot c^{n}+\text { terms of lower degree in } c,
$$

by the proof of (6.6). Considering the diagram

$$
\begin{gathered}
B^{*}-V^{*} \stackrel{i}{\rightarrow} B^{*} \\
\downarrow q^{\prime} \quad \downarrow q \\
X-U \stackrel{j}{\rightarrow} X
\end{gathered}
$$

we see that $i^{*} q^{*} \bar{w}_{m-n}=0$ by (6.1), hence $q^{*} j^{*} \bar{w}_{m-n}=0$ and $j^{*} \bar{w}_{m-n}=0$. By exactness in

$$
H_{c}^{m-n}(U) \rightarrow H^{m-n}(X) \stackrel{j^{*}}{\rightarrow} H^{m-n}(X-U),
$$

$\bar{w}_{m-n}$ is in the image of $H_{c}^{m-1}(U) \rightarrow H^{m-n}(X)$. That is, $A$ supports $\bar{w}_{m-n}$. Of course the same argument also works for $\bar{w}_{p}, p>m-n$.

(6.10) Corollary. Suppose that $p: B \rightarrow X$ is a bundle of $n$-spheres over a compact space $X$, and that $f: B \rightarrow R^{m}$ is equivariant with respect 
to the antipodal maps. The set $S \subset X$ of points $x$ such that $f(b)=0$ for some $b \in B_{x}$ supports every $\bar{w}_{p}, p \geqq m-n$.

If the base space $X$ is a compact connected manifold, then the set $S$ described in (6.10) has dimension at least $\operatorname{dim} X-p$ provided $\bar{w}_{p} \neq 0$ and $p \geqq m-n$. Since $S$ contains the support of $\bar{v}_{p}$ it follows from the Poincare duality theorem that $\operatorname{dim} S \geqq \operatorname{dim} X-p$.

We turn now to the index of $T: B \rightarrow B$. Our preceding results suggest that the co-index of a sphere bundle is a delicate invariant of the bundle. In contrast, the index of the bundle of unit tangent vectors to an $n$-manifold turns out to be $n-1$.

(6.11) Theorem. Suppose that $X$ is a compact differentiable $n$ manifold and that $p: B \rightarrow X$ is the bundle of unit tangent vectors to $X$. Then $\operatorname{ind}(T, B)=n-1$.

Proof. Since $S^{n-1} \subset B$, we know that ind $B \geqq n-1$. Suppose now ind $B \geqq n$; that is, suppose there exists an equivariant map $f: S^{n} \rightarrow B$. Then $\overline{\bar{f}}: P_{n} \rightarrow B^{*}$ induces $\bar{f}^{*}: H^{n}\left(B^{*}\right) \rightarrow H^{n}\left(P_{n}\right)$ which maps the class $c^{n}(B)$ of $(T, B)$ onto $c^{n}\left(S^{n}\right)$. From (6.2)

$$
c^{n}(B)=q^{*} w_{1} \cdot c^{n-1}+\cdots+q^{*} w_{n},
$$

where the $w_{i}$ are the Stiefel-Whitney classes of $X$. Then

$$
c^{n}\left(S^{n}\right)=\bar{f}^{*} q^{*} w_{1} \cdot c^{n-1}+\cdots+\bar{f}^{*} q^{*} w_{n} .
$$

We need now the $\mathrm{Wu}$ formulas for $w_{i}[16]$. That is, there exist classes $U_{i} \in H^{i}(X), 1 \leqq i \leqq n / 2$, with

$$
w_{j}=\sum S q^{j-i} U_{i}
$$

Hence $c^{n}\left(S^{n}\right)=\sum_{i+j+k=n} S q^{i} V_{j} \cdot c^{k}, \quad V_{j}=\bar{f}^{*} q^{*} U_{j}$. Fix now $j$ in the above term. If $V_{j}=0$ then all the terms $S q^{i} V_{j} \cdot c^{k}=0$ for that $j$. If $V_{j} \neq 0$, then $V_{j}=c^{j}$. Then

$$
\begin{aligned}
\sum_{i, k} S_{q}^{i} V_{j} \cdot c^{k}=\left(\sum_{i}\left\{\begin{array}{l}
j \\
i
\end{array}\right\}\right) c^{n} & \\
& \text { where }\left\{\begin{array}{l}
j \\
i
\end{array}\right\} \text { is the mod } 2 \text { binomial coefficient. }
\end{aligned}
$$

But $\sum_{i}\left\{\begin{array}{l}1 \\ i\end{array}\right\}=0(\bmod 2)$. It follows then that $c^{n}\left(S^{n}\right)=0$. But this is false, and the theorem is proved.

In particular, it follows from (3.9) that every equivariant map $S^{n-1} \rightarrow B$ is essential. In particular, the fibre $S^{n-1}$ is not contractible to a point in $B$. There is also the following restatement of (6.11).

(6.12) Suppose that $f$ is a continuous map of $S^{n}$ into a compact Rie- 
mannian n-manifold $X$. Then for some $x$ either $f(x)=f(-x)$ or for some $x, f(x)$ and $f(-x)$ are not joined by a unique geodesic arc of shortest length.

For suppose the theorem false. For each $x \in S^{n}$, let $F(x)$ denote the unit tangent vector to the unique geodesic of shortest length joining $f(x)$ to $f(-x)$, at the midpoint of the geodesic and pointing toward $f(-x)$. Then $F$ maps $S^{n}$ equivariantly into $V_{1}(X)$. But we have seen by (6.11) that this is impossible.

\section{REFERENCES}

1. A. Borel, La cohomologie mod 2 de certains espaces homogènes, Comment. Math. Helv. vol. 27 (1953) pp. 165-197.

2. K. Borsuk, Drei Sätze über die n-dimensionale euklidische Sphäre, Fund. Math. vol. 20 (1933) pp. 177-190.

3. D. G. Bourgin, On some separation and mapping theorems, Comment. Math. Helv. vol. 29 (1955) pp. 199-214.

4. H. Cartan and S. Eilenberg, Homological algebra, Princeton, Princeton University Press, 1956.

5. S. Eilenberg, Homology of spaces with operators. I, Trans. Amer. Math. Soc. vol. 61 (1947) pp. 378-417.

6. A. Grothendieck, Sur quelques points d'algèbre homologique, Tôhoku Math. J. vol. 9 (1957) pp. 119-220.

7. A. Heller, On equivariant maps of spaces with operators, Ann. of Math. vol. 55 (1952) pp. 223-231.

8. F. Hirzebruch, Neue Topologische Methoden in der Algebraischen Geometrie, Berlin, Springer-Verlag, 1956.

9. S. T. Hu, Homotopy theory, New York, Interscience, 1959.

10. L. Lyusternik and Schnirelmann, Topological methods in variational problems, Moscow, 1930.

11. J. P. Serre, Homologie singulière des espaces fibrés. Applications, Ann. of Math. vol. 54 (1951) pp. 425-505.

12. E. H. Spanier, Duality and S-theory, Bull. Amer. Math. Soc. vol. 62 (1956) pp. 194-203.

13. E. H. Spanier and J. H. C. Whitehead, The theory of carriers and S-theory, Algebraic geometry and topology, Princeton, Princeton University Press, 1957, pp. $330-360$.

14. N. Steenrod, The topology of fibre bundles, Princeton, Princeton University Press, 1951.

15. R. Thom, Espaces fibrés en sphères et carrés de Steenrod, Ann. Sci. Ecole Norm. Sup. vol. 69 (1952) pp. 109-182.

16. W. Wu, Classes caractéristiques et i-carrés d'une variêtê, C. R. Acad. Sci. Paris vol. 230 (1950) pp. 508-511.

17. C. T. Yang, Continuous functions from spheres to Euclidean spaces, Ann. of Math. vol. 62 (1955) pp. 284-292.

18. - On theorems of Borsuk-Ulam, Kakutani-Yamabe-Yujobo and Dyson. I, Ann. of Math. vol. 60 (1954) pp. 262-282.

19. - On theorems of Borsuk-Ulam, Kakutani-Yamabe-Yujobo and Dyson. II, Ann. of Math. vol. 62 (1955) pp. 271-283.

UNIVERSITY OF ViRGINIA 